\title{
The internal structure of asteroid (25143) Itokawa as revealed by detection of YORP spin-up $\star, \star \star$
}

\author{
S. C. Lowry ${ }^{1}$, P. R. Weissman ${ }^{2}$, S. R. Duddy ${ }^{1}$, B. Rozitis ${ }^{3}$, A. Fitzsimmons ${ }^{4}$, S. F. Green ${ }^{3}$, M. D. Hicks ${ }^{2}$, \\ C. Snodgrass ${ }^{5}$, S. D. Wolters ${ }^{3}$, S. R. Chesley ${ }^{2}$, J. Pittichová ${ }^{2}$, and P. van Oers ${ }^{6}$ \\ ${ }^{1}$ Centre for Astrophysics and Planetary Science, School of Physical Sciences (SEPnet), The University of Kent, \\ Canterbury, CT2 7NH, UK \\ e-mail: s.c.lowry@kent.ac.uk \\ 2 Jet Propulsion Laboratory, California Institute of Technology, Pasadena, CA 91109, USA \\ 3 Planetary and Space Sciences, Department of Physical Sciences, The Open University, Milton Keynes, MK7 6AA, UK \\ 4 Astrophysics Research Centre, Queens University Belfast, Belfast, BT7 1NN, UK \\ 5 Max Planck Institute for Solar System Research, Max-Planck-Str. 2, 37191 Katlenburg-Lindau, Germany \\ ${ }^{6}$ Isaac Newton Group of Telescopes, 38700 Santa Cruz de la Palma, Canary Islands, Spain
}

Received 3 September 2013 / Accepted 16 December 2013

\begin{abstract}
Context. Near-Earth asteroid (25143) Itokawa was visited by the Hayabusa spacecraft in 2005, resulting in a highly detailed shape and surface topography model. This model has led to several predictions for the expected radiative torques on this asteroid, suggesting that its spin rate should be decelerating.

Aims. To detect changes in rotation rate that may be due to YORP-induced radiative torques, which in turn may be used to investigate the interior structure of the asteroid.

Methods. Through an observational survey spanning 2001 to 2013 we obtained rotational lightcurve data at various times over the last five close Earth-approaches of the asteroid. We applied a polyhedron-shape-modelling technique to assess the spin-state of the asteroid and its long term evolution. We also applied a detailed thermophysical analysis to the shape model determined from the Hayabusa spacecraft.

Results. We have successfully measured an acceleration in Itokawa's spin rate of $\mathrm{d} \omega / \mathrm{d} t=(3.54 \pm 0.38) \times 10^{-8} \mathrm{rad} \mathrm{day}^{-2}$, equivalent to a decrease of its rotation period of $\sim 45 \mathrm{~ms} \mathrm{year}^{-1}$. From the thermophysical analysis we find that the centre-of-mass for Itokawa must be shifted by $\sim 21 \mathrm{~m}$ along the long-axis of the asteroid to reconcile the observed YORP strength with theory.

Conclusions. This can be explained if Itokawa is composed of two separate bodies with very different bulk densities of $1750 \pm 110 \mathrm{~kg} \mathrm{~m}^{-3}$ and $2850 \pm 500 \mathrm{~kg} \mathrm{~m}^{-3}$, and was formed from the merger of two separate bodies, either in the aftermath of a catastrophic disruption of a larger differentiated body, or from the collapse of a binary system. We therefore demonstrate that an observational measurement of radiative torques, when combined with a detailed shape model, can provide insight into the interior structure of an asteroid. Futhermore, this is the first measurement of density inhomogeneity within an asteroidal body, that reveals significant internal structure variation. A specialised spacecraft is normally required for this.
\end{abstract}

Key words. methods: observational - minor planets, asteroids: individual: (25143) Itokawa - techniques: photometric radiation mechanisms: thermal

\section{Introduction}

Asteroid (25 143) Itokawa is a relatively small near-Earth asteroid and its physical evolution is likely to be strongly affected by the Yarkovsky-O'Keefe-Radzievskii-Paddack (YORP) effect, which is a torque that can modify the rotation rates and spin-axis orientations of small bodies in the solar system. It is caused by the recoil effect from the anisotropic reflection and emission of solar radiation and thermal photons, respectively (Rubincam 2000). This process is responsible for many observed phenomena in asteroid science (Slivan 2002; Vokrouhlický et al. 2003; Ostro et al. 2006; Vokrouhlický \& Nesvorný 2008; Pravec et al. 2010), and was detected on the very small, fast spinning

* Based in part on observations collected at the European Southern Observatory, Chile, under programme ID: 185.C-1033.

$\star \star$ Table 2 is available at the CDS via anonymous ftp to cdsarc.u-strasbg.fr (130.79.128.5) or via

http://cdsarc.u-strasbg.fr/viz-bin/qcat?J/A+A/562/A48
near-Earth asteroid (54509) YORP (Lowry et al. 2007; Taylor et al. 2007). The effect has also been detected on asteroids (1862) Apollo and (1620) Geographos (Kaasalainen et al. 2007; Durech et al. 2008a), and with a tentative detection on asteroid (3103) Eger (Ďurech et al. 2012).

Itokawa is an important target for the study of the YORP effect as we can apply state-of-the-art thermophysical modelling to the detailed spacecraft shape model (Saito et al. 2006), to determine the expected YORP strength for the asteroid given its current orbital and spin-state properties. If the observed angular acceleration cannot be reconciled with theoretical predictions, then we can begin to explore other causes for the discrepancy. This may include inhomogeneous mass distributions within the body or non-uniform surface roughness, thus placing valuable constraints thereon (Scheeres \& Gaskell 2008). The potential for using an observed measurement of YORP to reliably probe the interior structure of an asteroid is unique among remoteobserving techniques and analysis methods. 
Table 1. Observational log and geometry for all optical photometry.

\begin{tabular}{lcccccccc}
\hline \hline Telescope & LC No. & $\begin{array}{c}\text { Date } \\
{[\mathrm{dd} / \mathrm{mm} / \mathrm{yy}]}\end{array}$ & $\begin{array}{c}R_{\mathrm{h}} \\
{[\mathrm{AU}]}\end{array}$ & $\begin{array}{c}\Delta \\
{[\mathrm{AU}]}\end{array}$ & $\begin{array}{c}\alpha \\
{[\mathrm{deg}]}\end{array}$ & $\begin{array}{c}\text { Aspect angle } \\
{[\mathrm{deg}]}\end{array}$ & $\begin{array}{c}\text { No. of data } \\
\text { points }\end{array}$ & $\begin{array}{c}\text { Phase offset } \\
\phi_{\text {YORP }} \pm 1-\sigma[\mathrm{deg}]\end{array}$ \\
\hline PAL60 & 1 & $22-24 / 08 / 01$ & 1.30 & 0.32 & 19.2 & 97.1 & 48 & $0.0 \pm<0.5$ \\
PAL60 & 2 & $22-25 / 09 / 01$ & 1.43 & 0.45 & 15.6 & 95.4 & 30 & $0.0 \pm<0.5$ \\
TMO & 3 & $27-30 / 01 / 04$ & 1.31 & 0.33 & 5.9 & 83.9 & 62 & $0.5 \pm<0.5$ \\
S60 & 4 & $24-25 / 01 / 07$ & 1.40 & 0.46 & 20.9 & 86.0 & 24 & $4.0 \pm<0.5$ \\
S90 & 5 & $10 / 12 / 09$ & 1.58 & 0.60 & 4.6 & 88.2 & 48 & $7.0 \pm<0.5$ \\
LT & 6 & $10 / 01 / 10$ & 1.51 & 0.58 & 20.0 & 87.4 & 99 & $9.0 \pm<0.5$ \\
LT & 7 & $09 / 12 / 12$ & 1.63 & 0.65 & 0.0 & 89.0 & 179 & $17.0 \pm<0.5$ \\
NTT & 8 & $14-16 / 12 / 12$ & 1.62 & 0.65 & 6.8 & 88.8 & 76 & $16.0 \pm<0.5$ \\
INT & 9 & $20 / 12 / 12$ & 1.61 & 0.69 & 11.4 & 88.7 & 321 & $15.5 \pm<0.5$ \\
PAL200 & 10 & $5-6 / 01 / 13$ & 1.57 & 0.69 & 6.8 & 88.8 & 36 & $15.0 \pm<0.5$ \\
\hline
\end{tabular}

Notes. All images were taken in either the broadband $V$ - or $R$-filter $\left(\lambda_{\mathrm{c}(V)}=550 \mathrm{~nm}, \lambda_{\mathrm{c}(R)}=657 \mathrm{~nm}\right)$. Telescope key: PAL60 - Palomar Observatory 60-inch Telescope (California, USA), TMO - Table Mountain Observatory (California, USA), S60 - Steward Observatory 60-inch Telescope (Arizona, USA), S90 - Steward Observatory 90-inch Bok Telescope (Arizona, USA), LT - 2 m Liverpool Telescope (La Palma, Spain), NTT European Southern Observatory $3.5 \mathrm{~m}$ New Technology Telescope (Chile), INT - $2.5 \mathrm{~m}$ Isaac Newton Telescope (La Palma, Spain), PAL200 Palomar Observatory $5 \mathrm{~m}$ Hale Telescope (California, USA).

Here we present results and analysis from a long-term photometric monitoring programme designed to detect changes in rotation rate that may be due to YORP. The structure of the paper is as follows: Sects. 2 and 3 describe the observational data that was acquired and how the rotational lightcurves were extracted and analysed to detect YORP accelerations. In Sect. 4 we present our thermophysical analysis as applied to the detailed spacecraft shape model, in order to provide a comparision of our results with theory. Section 5 provides a general discussion of the results, and their implications.

\section{Optical observations, and lightcurve extraction and modelling}

We monitored Itokawa between August 2001 and January 2013, using ground-based optical telescopes in Chile, the US and Europe (Table 1). The asteroid was observed at 10 different epochs for 1-3 nights each time (LC1-10). On each occasion time-series optical CCD imaging was obtained in either the broadband $V$ or $R$ filters. The aspect angle (angle between observer line-of-sight and the known rotation axis of the asteroid) changed little during the entire monitoring period, which can help to reduce measurement uncertainties in any detection of YORP. LC1-4 were included in a previous inconclusive attempt to detect YORP on Itokawa (Durech et al. 2008b). We followed up with new observations from 2009-2013 (LC5-10).

Bias subtractions and flat-fielding were performed in the usual manner. After this initial processing the images were then co-added to increase the signal-to-noise ratio and thus the quality of the extracted lightcurves. The images were aligned so that the background stars in each image appeared stationary in the field of view. These aligned images were then shifted according to the apparent rates of motion of the asteroid in order to produce a series of images in which the asteroid appeared stationary in the field. The images in each set were then co-added in groups. In the case of the data from the Isaac Newton Telescope (DS9 in Table 1), groups of twelve images were combined, while the group size was just four for the New Technology Telescope data (DS8). In general, the group size was kept small enough so that the total exposure time was less than $5 \%$ of the rotation period of the asteroid or $\sim 2100 \mathrm{~s}$, but large enough to produce an appreciable increase in the quality of the extracted lightcurve.
Aperture photometry was then performed to measure the brightness of the asteroid relative to background stars of constant brightness. The full width at half maximum (FWHM) of the seeing profile in each image was used to set the radius of the photometry aperture for the asteroid. This was normally measured using the background stars, although in some cases these were significantly trailed due to long exposure times and the use of non-sidereal tracking. In this case, the FWHM of the seeing profile was measured directly from the asteroid. Where trailing of background stars was evident, rectangular photometry apertures were preferred for the comparison stars. This minimizes any sky-background contribution, which can reduce the quality of the photometry. The rectangular apertures were centred on the middle of the star trail, and their length and direction in the image were calculated from the exposure time and rates of motion of the asteroid. The width of the aperture is set to be equal to the FWHM of the seeing profile measured from the asteroid. This method improves the quality of the extracted lightcurves, especially in those cases where the background stars might be faint. Instrumental magnitudes for the background comparison stars were measured and a weighted average taken to ensure that variations in stellar brightness for the fainter stars had minimal affect on the asteroid rotational lightcurves.

\subsection{Model-lightcurve generation from polyhedron shape models}

Upon extraction of the rotational lightcurves, each datapoint was light-time corrected. In the case of Itokawa this was typically on the order of several minutes. This step is crucial to ensure accurate measurements of the rotational phase offset between the artificial and observed lightcurves, as an error of one minute can introduce an uncertainty of 0.5 degrees in rotation phase for this asteroid. The light-travel time, the direction vectors of the asteroid from the sun and the observer, and the topocentric positions, were calculated using the JPL HORIZONS online system.

Artificial lightcurves were generated using a convex hull of the Itokawa shape model developed by Gaskell (2008). The Gaskell model consists of several hundred thousand facets and is highly detailed. However, since the rotational lightcurve is the result of changes in the area projected towards the observer, the extreme detail of this model is unnecessary. Therefore the model was scaled down to a convex hull of only 2436 facets as has been 
done in previous studies (Ďurech et al. 2008a), and is quite sufficient for our purposes. This has the advantage that it significantly speeds up the generation of the artificial lightcurves.

The pole orientation of the asteroid as measured by the spacecraft (Demura et al. 2006) was used to model its rotation in space. The rotational phase $\theta$ of the shape model is calculated for each data point using,

$\theta=\omega_{0}\left(t-T_{0}\right)+(v / 2)\left(t-T_{0}\right)^{2}$

where $\omega_{0}$ is the initial angular velocity of the asteroid, $t$ is the time of the observation, $T_{0}$ is a fixed arbitrary time related to the initial orientation of the asteroid, and $v(=\mathrm{d} \omega / \mathrm{d} t)$ is the rate of change of angular velocity with time or the YORP strength or acceleration. A ray-tracing algorithm was used to determine the angles between each facet normal and both the Sun and observer. The scattering model employed was a simple combination of a Lambert surface and the Lommel-Seeliger model (Kaasalainen et al. 2001). The flux from each facet was then summed to produce the expected brightness of the asteroid for each datapoint and converted to a magnitude.

The artificial and observed lightcurves are then placed on the same relative scale. This was achieved by first subtracting the average brightness from the artificial and observed lightcurves so that the amplitudes oscillated about zero magnitudes. Secondly, a range of small vertical shifts was applied to the artificial lightcurves and the $\chi^{2}$ difference between the artificial and observed lightcurves calculated. This metric was employed throughout the analysis to determine the quality of the fit between the artificial and observed lightcurves. The vertical shift in brightness corresponding to the lowest $\chi^{2}$ value was then applied to ensure that the lightcurves had the same brightness scale.

We then determine the initial orientation of the asteroid in space. As described earlier this is related to the parameter $T_{0} . T_{0}$ can be assigned arbitrarily and an additional rotation applied to the shape model. However, for the purposes of our analysis, $T_{0}$ was assigned such that the artificial lightcurves were perfectly aligned with the lightcurves observed in August and September of 2001 (i.e. LC1+2 in Table 1). This was done by creating artificial lightcurves for the August and September 2001 data using a range of $T_{0}$ values separated by approximately half-degree intervals. The best $T_{0}$ was found to be 2452143.4815 (on August 21 st, $2001 \mathrm{UT}$ ). All subsequent models were advanced from this initial $T_{0}$. We can then incorporate a constant rotation period for the model, or a rotation period that is varying linearly with time.

\section{Measuring the observed YORP strength}

We adopted two different strategies for detecting and measuring YORP from the observational lightcurve data. The first involves the measurement of rotation phase offsets between the observed lightcurves and artificial lightcurves generated using the Hayabusa shape model with a fixed sidereal rotation period. A linear increase in rotation rate due to YORP causes a quadratic increase in rotational phase offset $\phi$ with time $t$,

$\phi=(v / 2)\left(t-T_{0}\right)^{2}+\left(\omega_{0}+\epsilon\right)\left(t-T_{0}\right)$

where $\epsilon$ is the difference between the estimated rotation rate and the actual rotation rate $\omega_{0}$, at time zero $T_{0}$. Any uncertainty in the initial rotation rate used to advance the model - that is any non-zero value for $\epsilon$ - introduces a linear increase in phase offset with time, easily distinguished from the quadratic phase offset progression due to YORP. This is an iterative process that

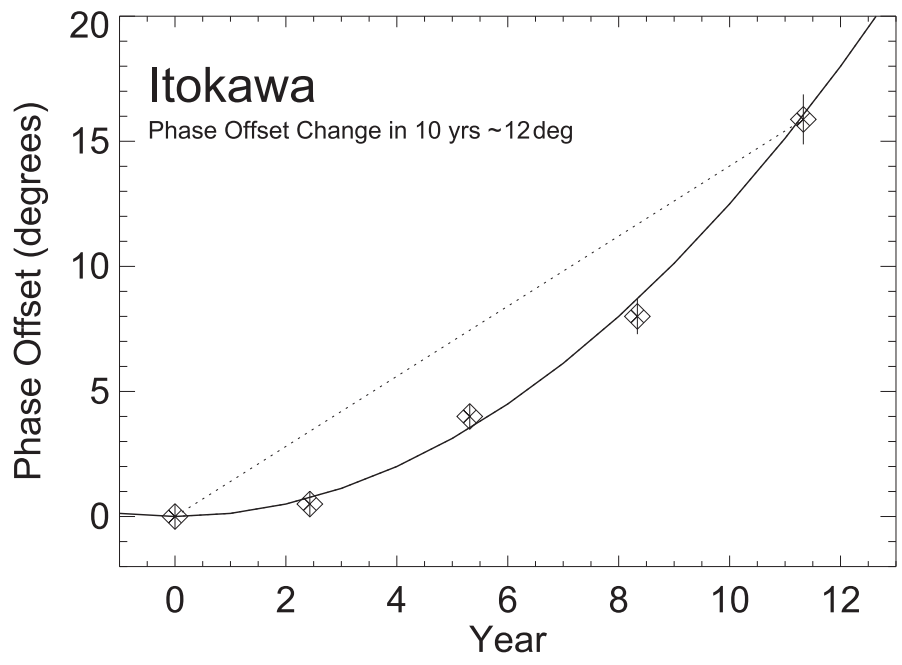

Fig. 1. Rotational phase changes $(\phi)$ in Itokawa's lightcurves observed from August/September 2001 to January 2013. The strong quadratic temporal variation of $\phi$ is perfectly consistent with YORP-induced rotational acceleration. The solid curve is the best-fit quadratic curve, and the dotted line connects the first and last data points, to emphasize the deviation from a straight line profile.

also allows us to determine any YORP acceleration or deceleration that may be present. We chose as our starting conditions an initial rotation period of $12.13245 \mathrm{~h}$, consistent with previous studies (Durech et al. 2008b). Once the artificial lightcurves were generated for each observed lightcurve with this starting period, we measured the rotational phase offsets between them. This was done for each lightcurve separately, by applying an incremental phase offset to the artificial lightcurve and calculating the $\chi^{2}$ value. We sweep through a suitable range of phase offsets until the $\chi^{2}$ value is minimized. The error-bars, as listed in Col. 9 of Table 1, are the formal $1-\sigma$ uncertainties from the $\chi^{2}$ fitting process. Measurement of the phase offsets indicated a YORP strength of $(3.28 \pm 0.49) \times 10^{-8} \mathrm{rad} \mathrm{day}^{-2}$ and an initial rotation period of $12.13237 \mathrm{~h}$. Repeating this procedure with the new input rotation period produced a YORP strength of $(3.19 \pm 0.41) \times 10^{-8} \mathrm{rad} \mathrm{day}^{-2}$ and an initial rotation period of $12.132369 \mathrm{~h}$. We repeated the procedure until no further significant variation was observed. The final YORP strength measured via this method was $(3.27 \pm 0.29) \times 10^{-8}$ rad day $^{-2}$ with an initial rotation period at $T_{0}$ of $12.132371 \pm\left(6 \times 10^{-6}\right) \mathrm{h}$ (Fig. 1). Previous analysis had suggested that a fixed rotationperiod model fitted all data between 2001 and 2008 (Durech et al. 2008b). The fixed-period model fit the data reasonably well until 2009 when a significant offset in phase between the artificial and observed lightcurves became very clear (Figs. 1 and 2). This offset in phase increased further between 2009 and 2013 indicating that the rotation rate was not fixed but was changing linearly with time, completely consistent with YORP.

The second procedure involves producing artificial lightcurves over a large grid of initial sidereal rotation periods and YORP values and measuring the $\chi^{2}$ value at each iteration, i.e. we allow the shape model rotation rate to change linearly with time. In this way we can determine the relationship between the initial rotation period used to advance the model and the observed angular acceleration. We conducted a search over the rotation period range $12.13238 \pm 10^{-4} \mathrm{~h}$ in intervals of $2.5 \times 10^{-8} \mathrm{~h}$ and with various YORP strengths in the range $(5 \pm 5) \times 10^{-8} \mathrm{rad} \mathrm{day}^{-2}$, and at intervals of 

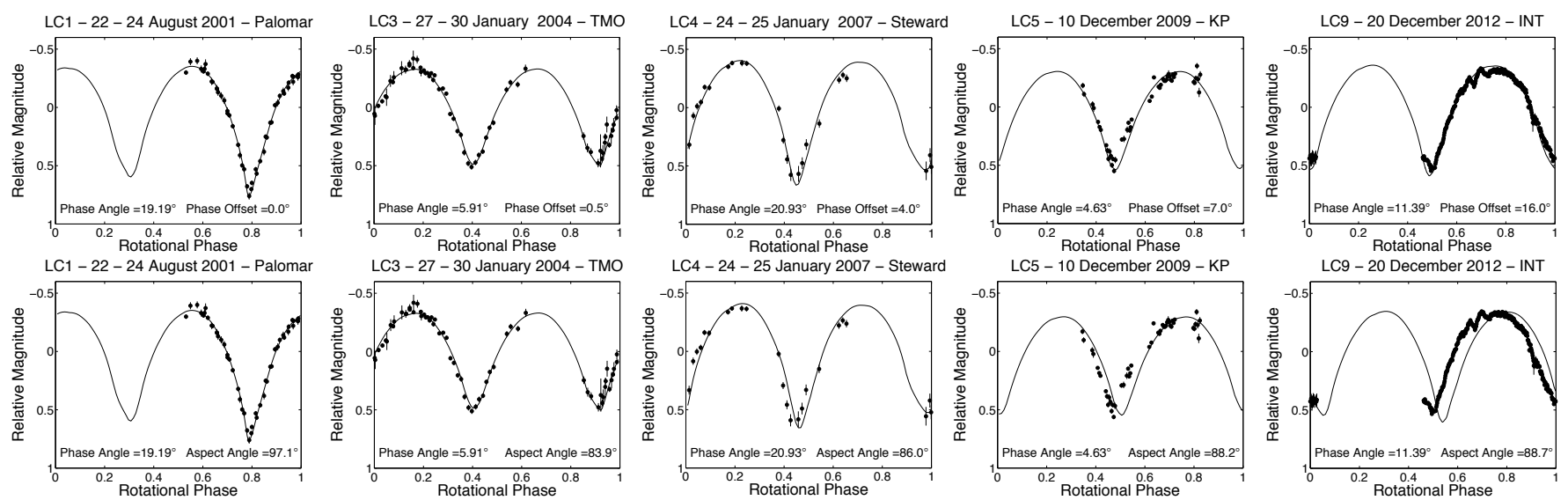

Fig. 2. A representative sample of lightcurves generated using the convex model of Itokawa. The solid line represents the artificial lightcurves and the dots are the observed magnitude data points. In the upper panels, the model has been advanced with a rotation period changing due to YORP. In the the lower panels, the lightcurves are generated with a fixed period model. It is clear that there is a progressive increase in the rotation phase offset between the artificial lightcurves and observed data points when a fixed period model is used. Incorporating a linear change in rotation rate, consistent with YORP, corrects the rotational phase of the artificial lightcurves (also see Fig. 1).

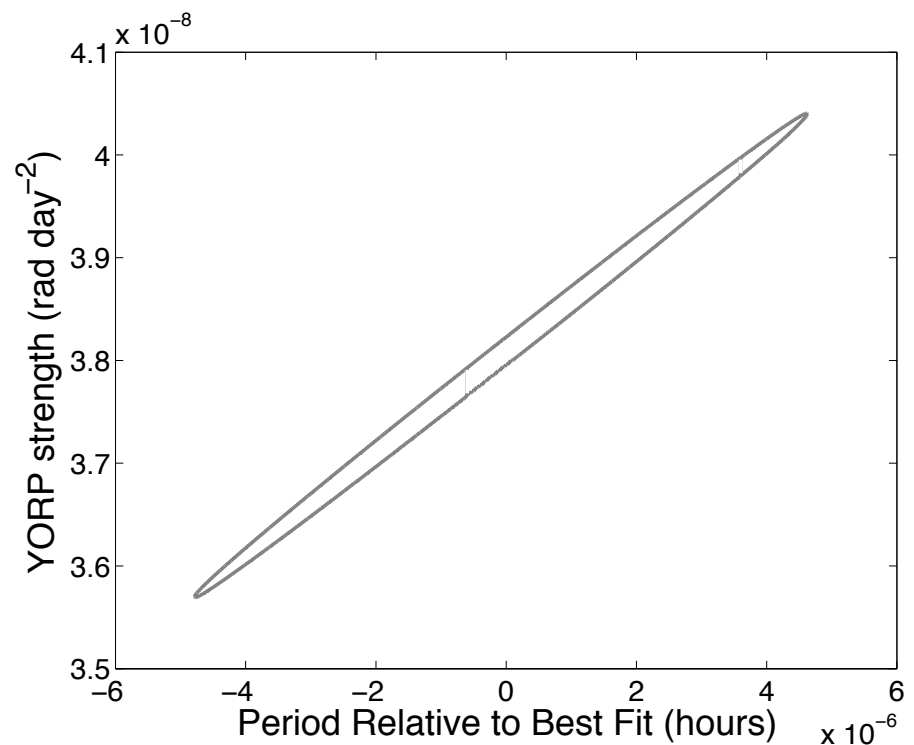

Fig. 3. $1-\sigma$ uncertainty ellipse for the optimum initial rotational period and YORP strength of the spin-state model to produce the best fit between artificial lightcurves and observations. YORP-spin up is required, with the best-fit obtained using $12.1323789 \mathrm{~h}$ for the sidereal rotation period at $T_{0}$ and a corresponding YORP strength of $3.81 \times 10^{-8}$ rad day $^{-2}$. From the $1-\sigma$ uncertainty ellipse we determine the uncertainty in period to be $4.7 \times 10^{-6} \mathrm{~h}$ and the corresponding uncertainty in YORP to be $0.24 \times 10^{-8} \mathrm{rad} \mathrm{day}^{-2}$. Note that in this case where we have a spacecraft shape model with a well-determined pole solution, the uncertainty in the fitted initial period becomes the dominating influence on the uncertainty in the measured YORP value.

$10^{-11} \mathrm{rad} \mathrm{day}^{-2}$. Our best-fit parameters found with this method are $12.1323789\left( \pm 4.7 \times 10^{-6}\right) \mathrm{h}$ for a YORP strength of $(3.81 \pm 0.24) \times 10^{-8} \mathrm{rad} \mathrm{day}^{-2}$ (see Fig. 3). This is consistent with the first method above at the $1-\sigma$ level.

For the subsequent analysis we adopt the average YORP value from the two methods of $v=(3.54 \pm 0.38) \times 10^{-8} \mathrm{rad} \mathrm{day}^{-2}$, which is equivalent to a decrease of Itokawa's rotation period of $45.4 \pm 4.9 \mathrm{~ms} \mathrm{year}^{-1}$.

\section{Thermophysical analysis and measured density inhomogeneity}

The observed rotational acceleration (i.e. YORP spin-up) is contrary to previous theoretical YORP studies, which predict strong rotational deceleration acting on Itokawa (i.e. YORP spin-down). In particular, studies based on the Hayabusa-derived shape models predicted rotational accelerations (Scheeres et al. 2007; Breiter, et al. 2009) of ( -5.5 to $-2.0) \times 10^{-7} \mathrm{rad} \mathrm{day}^{-2}$, which differ significantly from our observed value of $3.54 \times 10^{-8} \mathrm{rad} \mathrm{day}^{-2}$. These predictions were also inconsistent with an upper limit of $|v|<1.5 \times$ $10^{-7} \mathrm{rad} \mathrm{day}^{-2}$ that was derived from existing light-curve observations in 2008 (Durech et al. 2008b). To explain the inconsistency, it was suggested that a non-uniform internal mass distribution that shifted the centre-of-mass (COM) away from the "centre-of-figure" towards the "head" of Itokawa could be a possible cause (Scheeres \& Gaskell 2008). Other theoretical work indicated that the YORP effect can be extremely sensitive to unresolved shape features (Statler 2009), the shape model resolution (Breiter, et al. 2009), and surface roughness (Rozitis \& Green 2012), such that the error in any prediction could be very large.

We determined a theoretical YORP value for this asteroid by applying the advanced thermophysical model (ATPM; Rozitis \& Green 2011, 2012, 2013) to the 49,152-facet spacecraft shape model of Itokawa (Gaskell 2008). Assuming a moment of inertia, $I_{Z}$, of $7.77 \times 10^{14} \mathrm{~kg} \mathrm{~m}^{2}$ (Breiter et al. 2009) along with a moderately rough surface at $\mathrm{cm}$ scales (Ostro et al. 2004; Müller et al. 2005) with a uniform spatial distribution, the ATPM predicts a rotational acceleration of $-1.80 \times 10^{-7} \mathrm{rad} \mathrm{day}^{-2}$, consistent with previous determinations. A COM shift can reconcile our YORP model with the observed value, which we can determine by combining the ATPM with the methodology used for calculating such COM offsets (Scheeres \& Gaskell 2008). Possible new locations of the COM in Itokawa's $x-y$ plane exist along lines defined by

$T_{\mathrm{CM}}=T_{\mathrm{CF}}-F_{\mathrm{Y}} \Delta x+F_{\mathrm{X}} \Delta y$

where $\Delta x$ and $\Delta y$ are the COM offsets from the "centre-offigure" in Itokawa's body-fixed $x-y$ plane (n.b. distance from the centre-of-figure is $\left.\Delta r=\left(\Delta x^{2}+\Delta y^{2}\right)^{0.5}\right), F_{X}$ and $F_{Y}$ are the overall 


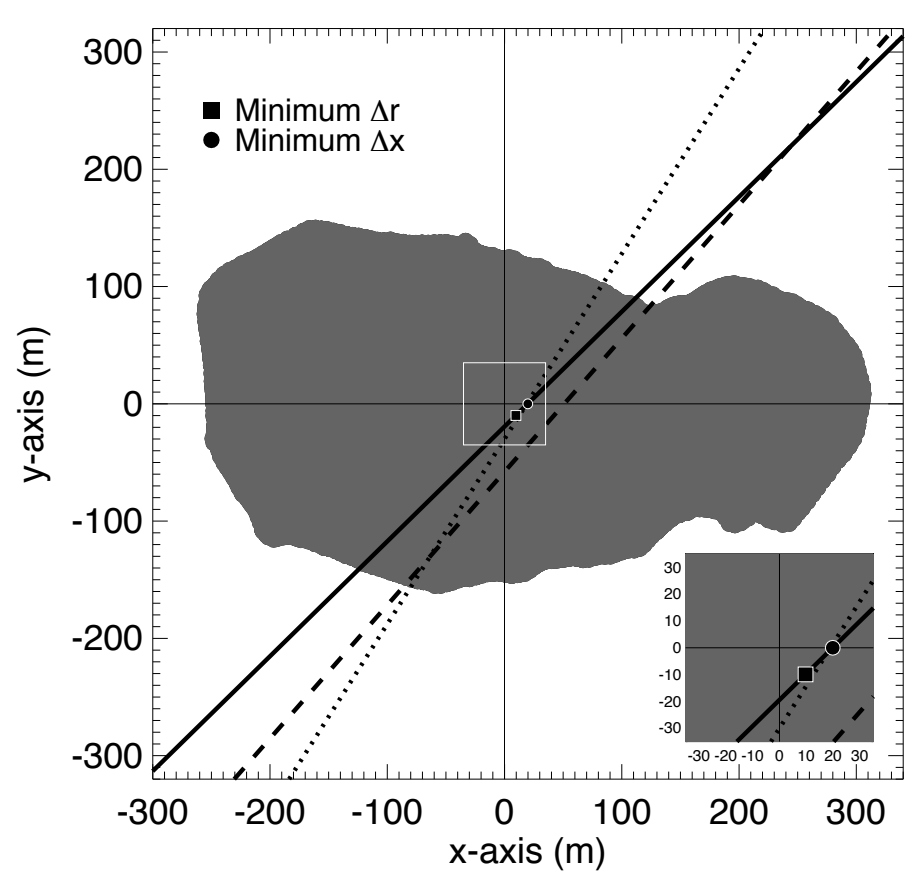

Fig. 4. Projection in the $x-y$ plane of Itokawa's shape model and possible new locations of the centre-of-mass to reconcile the observed YORP effect with theory. Assuming a moderately rough surface with a uniform spatial distribution, the ATPM calculates that the COM must exist somewhere along the solid line to reproduce the observed YORP rotational acceleration of $3.54 \times 10^{-8} \mathrm{rad} \mathrm{day}^{-2}$. The location with the minimum required offset from the centre-of-figure is shown by the black square $(\sim 14 \mathrm{~m}$ displacement), and the black circle shows the location if the offset is just along the $x$-axis ( $\sim 21 \mathrm{~m}$ displacement). Locations further along the line are less probable as they require greater and more unusual bulk density inhomogeneities to produce the larger offsets. The ATPM calculates the YORP rotational acceleration line with shadowing and global self-heating effects included. For comparison purposes, the zero YORP rotational acceleration lines with none of these effects included (Scheeres \& Gaskell 2008), and with only shadowing included (Breiter et al. 2009), are plotted as the dotted and dashed lines respectively. As demonstrated in Rozitis \& Green (2013), if global self-heating effects are neglected then YORP predictions are generally more accurate if shadowing is also not included. This explains why the calculations by Scheeres \& Gaskell (2008) are similar to ours as shown here.

photon force components acting on Itokawa in the same frame of reference, $T_{\mathrm{CM}}$ (i.e. $T_{\mathrm{CM}}=I_{Z} v$ ) is the inferred YORP torque acting about Itokawa's COM, and $T_{\mathrm{CF}}$ is the calculated YORP torque acting about Itokawa's centre-of-figure. This approach requires a minimum COM offset from the centre-of-figure, $\Delta r$, of $\sim 14 \mathrm{~m}$, or an offset, $\Delta x$, of $\sim 21 \mathrm{~m}$ if the offset is just along the $x$-axis where Itokawa's "body" and "head" are approximately aligned (see Fig. 4).

When surface roughness is included in the predictions then the thermal-IR beaming effect it induces has the tendency to dampen the YORP rotational acceleration on average but can add uncertainties of the order of several tens of per cent if the roughness is allowed to vary across the surface (Rozitis \& Green 2012). In this work, the unresolved surface roughness is described in terms of each shape facet containing a fractional coverage, $f_{R}$, of hemispherical craters, with the remaining fraction $\left(1-f_{R}\right)$, representing a smooth flat surface. The hemispherical crater is a simple way to accurately reproduce the thermal-IR beaming effect (i.e. re-radiation of absorbed sunlight back towards the Sun) produced by a range of surface roughness morphologies and spatial scales, and has been verified by

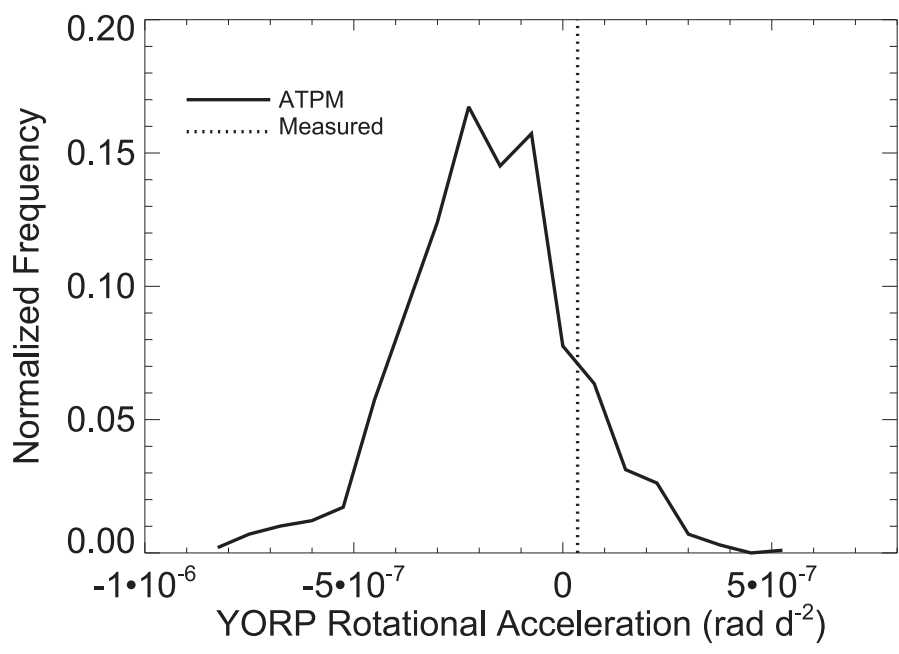

Fig. 5. Distribution of YORP rotational acceleration values acting on Itokawa predicted by ATPM for different patchy surface roughness distributions (solid line) compared with the observed value (dotted line).

application to lunar data (Rozitis \& Green 2011). These spatial scales start at the thermal skin depth $(\sim 1 \mathrm{~cm})$ and range up to the facet size of the shape model used ( $\sim 4 \mathrm{~m}$ in this case). Previous thermophysical modelling of thermal-infrared observations of Itokawa indicate that the surface is rough at these spatial scales but the distribution is unknown (Müller et al. 2005). Radar circular polarisation ratios also give an indication of an asteroid's wavelength-scale roughness (Ostro et al. 2002; Benner et al. 2008), and Itokawa's disk-integrated ratio at $3.5 \mathrm{~cm}, \mu_{\mathrm{C}}=0.47 \pm 0.04$, is significantly larger than that at $12.6 \mathrm{~cm}, \mu_{\mathrm{C}}=0.26 \pm 0.04$ (Ostro et al. 2004; Nolan et al. 2013). This indicates that most of the surface roughness occurs at the cm-scale, and won't effectively be described in the highest resolution shape model of Itokawa ( $\sim 3$ million facets) as it has a facet size of $0.5 \mathrm{~m}$ (Gaskell et al. 2008). These spatial scales are also much larger than the $<1 \mathrm{~mm}$ photometric roughness that is inferred from optical scattering (e.g. Hapke 1981; Hapke \& Wells 1981), and therefore we are unable at present to determine the real distribution of surface roughness for Itokawa from any kind of observation.

To assess the impact of potential surface roughness variations on the range of theoretical YORP values that may be possible, we performed a Monte Carlo analysis where the roughness was allowed to vary in a patchy way but still had the same value when averaged across the surface. To generate a patchy surface roughness distribution, the surface of Itokawa was divided into 10 randomly assigned areas that each have their own unique degree of roughness, which was also randomly chosen from a normal distribution with a mean value and standard deviation of $f_{R}=0.50 \pm 0.08$. The model reflected and emitted photon torques from each shape facet were adjusted according to the shape facet's individual roughness fraction, which were then summed across the surface to give the overall YORP torque acting on Itokawa, and hence the YORP rotational acceleration when divided by Itokawa's moment of inertia. Based on 1000 trials, the distribution of predicted YORP rotational acceleration had a mean value and standard deviation of $(-1.80 \pm 1.96) \times 10^{-7} \mathrm{rad} \mathrm{day}^{-2}$ (Fig. 5), which also encompasses the shape sensitivity range highlighted by previous studies (Scheeres et al. 2007; Breiter et al. 2009). In 16.5\% of these trials, a YORP spin-up was predicted. However, the roughness distributions that produce YORP rotational acceleration values 

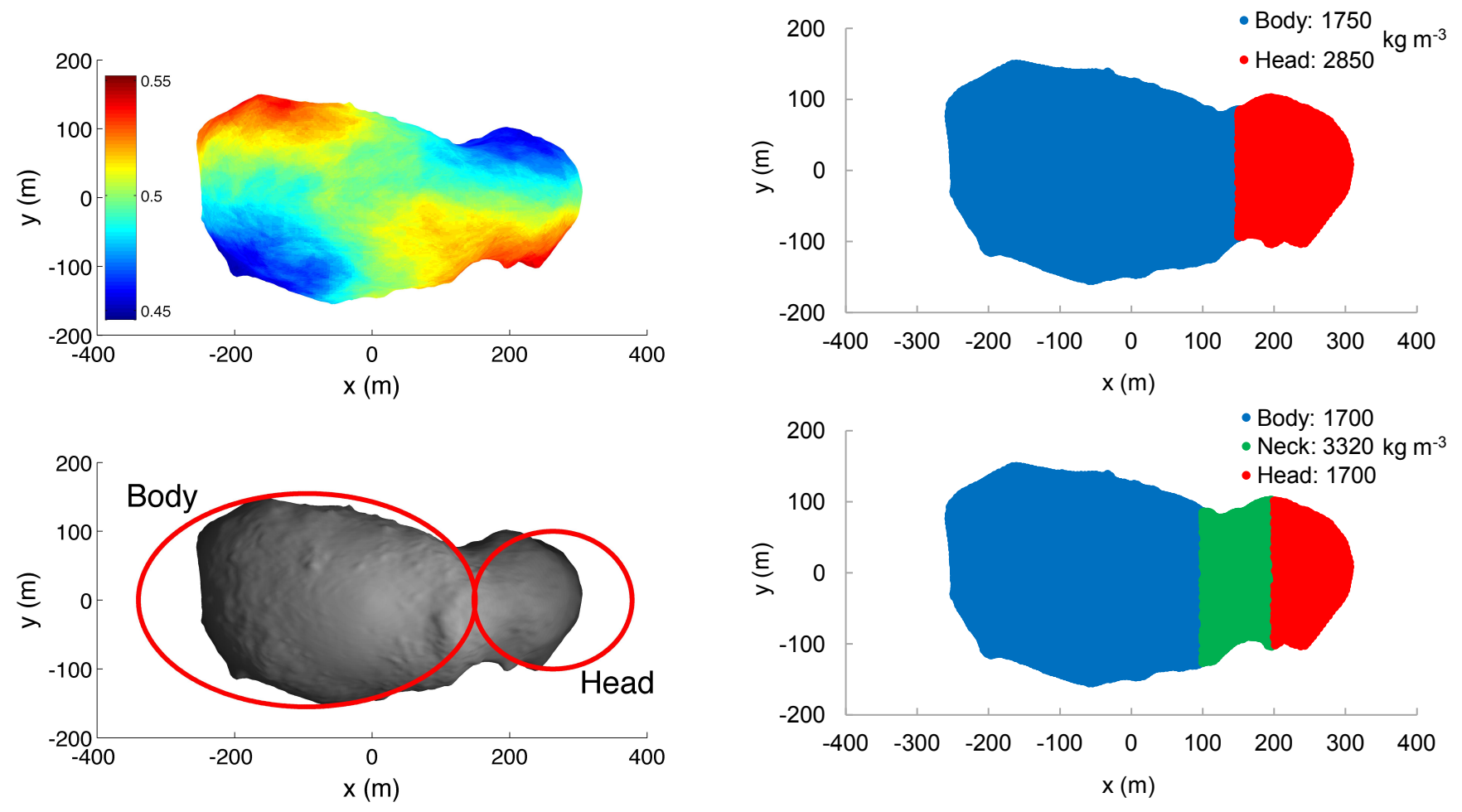

Fig. 6. Various stages of the thermophysical analysis used to attempt to reconcile the observed YORP acceleration with theoretical determinations. The pole-on shape model renderings were determined from imaging data from the Hayabusa spacecraft (Gaskell 2008), and highlight Itokawa's "bi-lobed" appearance (Demura et al. 2006). Upper Left Panel - Average surface roughness distribution of Itokawa clones that produce a YORP rotational acceleration (or YORP spin-up). The roughness scale ranges from 0.45 (blue) to 0.55 (red). Lower Left Panel - Regular ellipsoids that interface at $x \sim 150 \mathrm{~m}$, with relative dimensions chosen for consistency with Scheeres \& Gaskell (2008), which are loosely based on values from Demura et al. (2006). A density differential between the head and body can explain the COM offset. Upper Right Panel - Use of the true shape model with the interface between "body" and "head" regions of different densities at $x=150 \mathrm{~m}$. Lower Right Panel - A compressed "neck" region of higher density located between the "body" and "head" to explain the measured COM offset. See Sect. 4 for details.

similar to that observed have an artificial appearance that maximises the YORP spin-up and does not correspond with any geological features (Fig. 6). This shows that an asymmetric roughness distribution cannot alone be responsible for the observed YORP spin-up. Accounting for the YORP rotational acceleration uncertainty resulting from possible and realistic roughness distributions leads to potential COM offsets of $\Delta r=14 \pm 7 \mathrm{~m}$ or $\Delta x=21 \pm 12 \mathrm{~m}$ (Fig. 7).

A COM offset along the $x$-axis towards Itokawa's "head" strongly implies that it has a higher bulk density than the "body" (Scheeres \& Gaskell 2008). Approximating Itokawa's shape as two ellipsoids with dimensions of $490 \times 310 \times 260 \mathrm{~m}$ (i.e. "body") and $230 \times 200 \times 180 \mathrm{~m}$ (i.e. "head") resting on each other (Scheeres \& Gaskell 2008; Demura et al. 2006) (Fig. 6) allows the COM offset to be calculated as a function of the densities of the "body" and "head" (Fig. 8). $\Delta x=21 \pm 12 \mathrm{~m}$ results in bulk densities of $1810 \pm 80 \mathrm{~kg} \mathrm{~m}^{-3}$ and $2620 \pm 370 \mathrm{~kg} \mathrm{~m}^{-3}$, for the "body" and "head" respectively. The overall bulk density remains at $1950 \mathrm{~kg} \mathrm{~m}^{-3}$, in order to be consistent with the spacecraft-derived value from Abe et al. (2006b). The mass, and hence density of Itokawa was derived by Abe et al. from the measured acceleration of the spacecraft, determined using laser ranger data and an assumption of uniform density. In theory, any non-uniformity of density would have an effect on the spacecraft's trajectory when close to the asteroid. However, Hayabusa did not orbit Itokawa and the mass was derived from a single descent between 1.4 and $0.8 \mathrm{~km}$. Significant non-gravitational forces (from solar radiation pressure and thrusters) had also to be taken into account, resulting in a precision in the derived mass

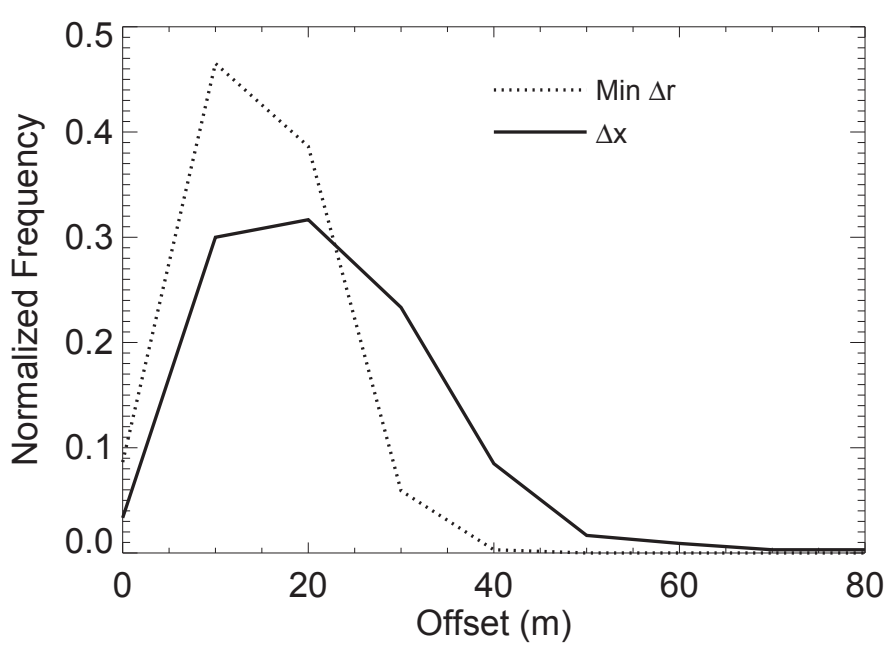

Fig. 7. Distribution of possible centre-of-mass offsets in terms of minimum distance from the centre-of-figure, $\Delta r$ (dotted line), and distance along the $x$-axis only, $\Delta x$ (solid line), derived using the results of the Monte Carlo analysis shown in Fig. 5. Itokawa clones with unrealistic surface roughness distributions (i.e. highly assymetric as in Fig. 6 or its transpose) are excluded.

of $5 \%$. It is impossible to determine if a density inhomogeneity could be determined from such limited measurements and there is no mention of this possibility in the paper. Our measurement 
S. C. Lowry et al.: YORP spin-up detected on asteroid Itokawa

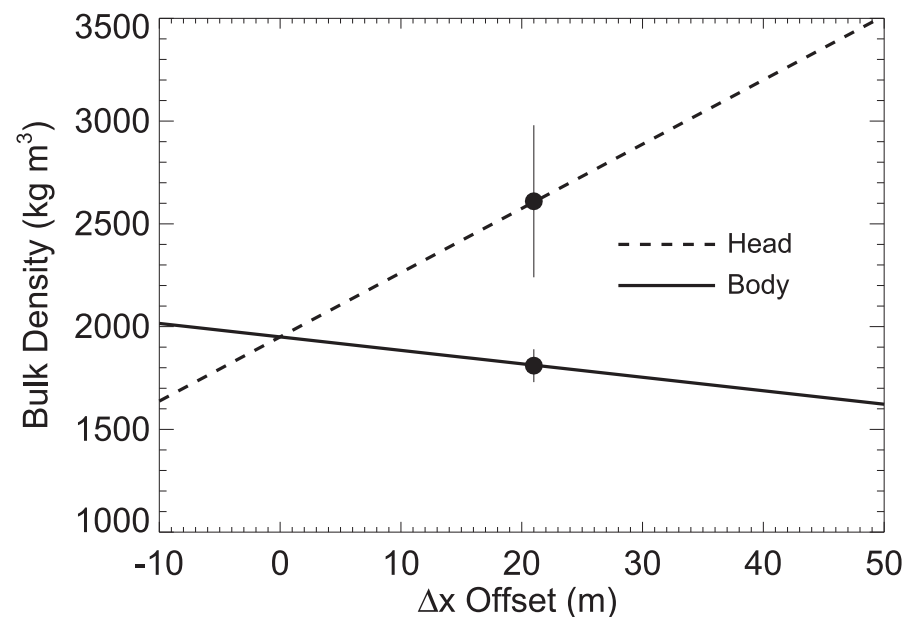

Fig. 8. Bulk density of Itokawa's "body" and "head" as a function of centre-of-mass offset $(\Delta x)$ along the $x$-axis. The overall bulk density of Itokawa remains at $1950 \mathrm{~kg} \mathrm{~m}^{-3}$, as determined from the Hayabusa spacecraft (Abe et al. 2006b).

of a significant density inhomogeneity is therefore not inconsistent with the Hayabusa study.

The ellipsoid approach is not an optimal representation of Itokawa so we repeat the calculations after simply dissecting the asteroid at $x=150 \mathrm{~m}$ as shown. The new density values are $1750 \pm 110 \mathrm{~kg} \mathrm{~m}^{-3}$ and $2850 \pm 500 \mathrm{~kg} \mathrm{~m}^{-3}$. The measured COM offsets, and thus the density difference between "body" and "head", are therefore not overly sensitive to the precise relative dimensions of the ellipses or any slight offset in the relative orientation between them. The derived bulk density difference between the two components of Itokawa is comparable to that seen between the primary $\left(1970 \pm 240 \mathrm{~kg} \mathrm{~m}^{-3}\right)$ and secondary $\left(2810+820 /-630 \mathrm{~kg} \mathrm{~m}^{-3}\right)$ of the $(66391) 1999 \mathrm{KW} 4$ binary system (Ostro et al. 2006).

We extended our analysis to see if the merging of two bodies of equal density, causing a localised compression around the contact interface or "neck" region, could explain the apparent COM offset (Figs. 6 and 9). Using Itokawa's shape model and assuming a uniform density we determined the mass distribution (which is also equal to the volume distribution) along Itokawa's $x$-axis. We find the neck to be narrowest at $+150 \mathrm{~m}$, which we assume to be the centre of the neck (Fig. 9, upper panel). To determine possible centre-of-mass (COM) offsets we varied the width and density of the neck, and the density of the remaining body is adjusted accordingly to ensure a constant mass for Itokawa. We represent the neck density as a multiple of the overall bulk density (e.g. $x 1.7$ means $1950 \mathrm{~kg} \mathrm{~m}^{-3} \times 1.7=3315 \mathrm{~kg} \mathrm{~m}^{-3}$ ), and this multiple ranges from 1.1 to 1.7 . (Fig. 9, lower panel). Multiples greater than 1.7 are not likely to be feasible as they give the neck a density greater than that of meteorites associated with S-type asteroids. A neck width of $\sim 100 \mathrm{~m}$ would be required to produce a nominal COM offset of $\sim 21 \mathrm{~m}$ when a neck density multiple of 1.7 is assumed. Neck widths greater than $\sim 50$ m seem unrealistic given that the head itself is merely $\sim 150 \mathrm{~m}$ in diameter. Furthermore, typical bulk porosities for S-type asteroids are between 20-40\%. Accounting for even the conservative lower end of this range sets a more realistic density multiple upper limit of $x 1.36$. The COM offset for a $50 \mathrm{~m}$ neck with this densitymultiple would be just $4.6 \mathrm{~m}$. For the range of plausible neck dimensions and densities, the neck concentration explanation can only realistically explain COM offsets less than $\sim 5 \mathrm{~m}$.
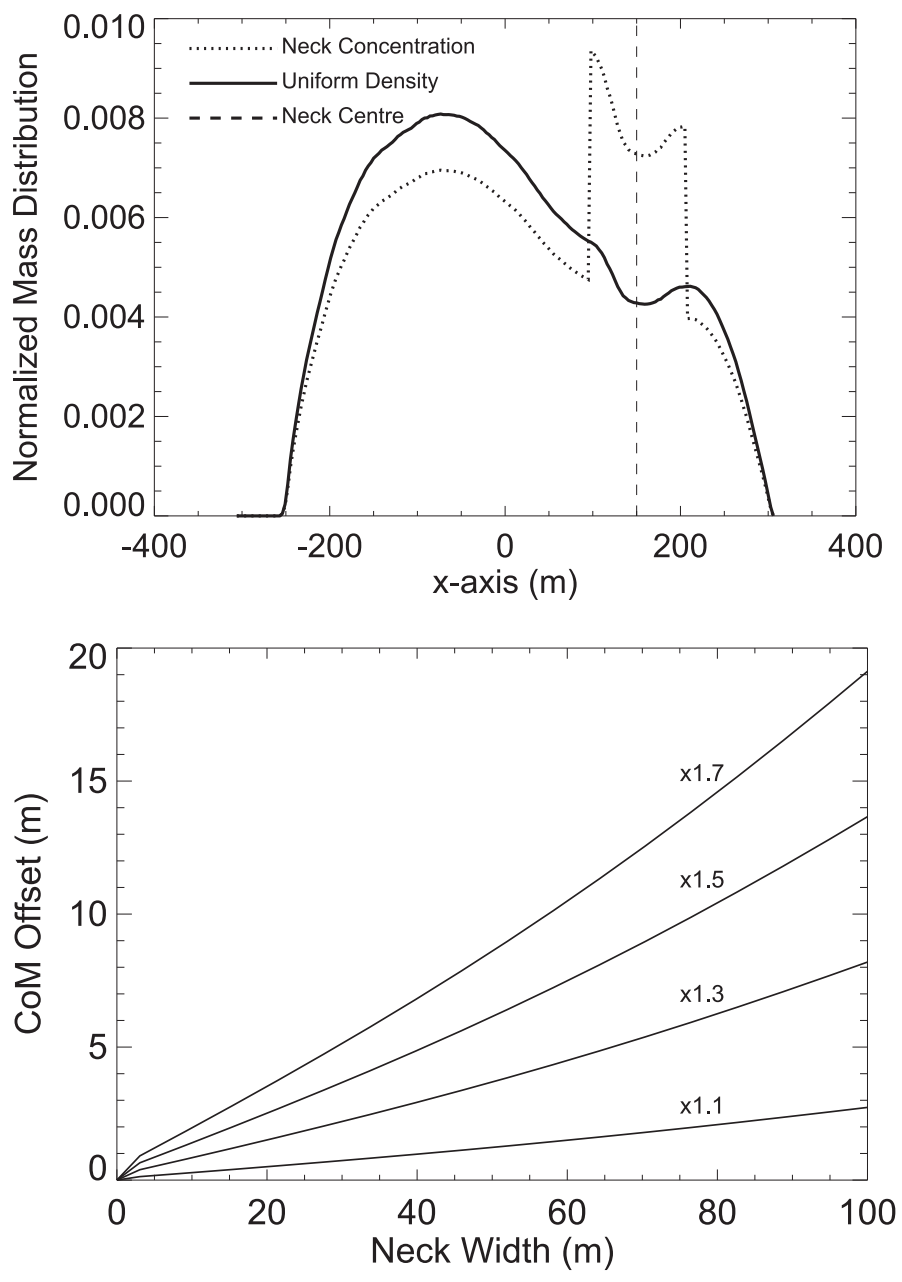

Fig. 9. Analysis of a compressed "neck" region between the "body" and "head" of Itokawa to explain the centre-of-mass (COM) offset. Top Panel - The normalised mass distribution along Itokawa's $x$-axis. The solid line is for uniform density, and the dotted line is for the extreme case of the same overall mass but with a neck of diameter $100 \mathrm{~m}$ and density defined by a neck-density multiple of 1.7 times the overall bulk density. The vertical dashed line indicates the location of the centre of the neck defined by the minimum in the mass distribution. Bottom Panel - The COM offset as a function of neck width and neck-density multiple. A multiple of 1.7 corresponds to a density equal to that of solid meteorites associated with S-type asteroids.

\section{Discussion}

We speculate on the various scenarios that may explain the apparent COM shift and density inhomogeneity. Such scenarios include:

a) The merging of two bodies of equal density, causing a localised compression around the contact interface or "neck" region. This scenario is consistent with findings from the Hayabusa spacecraft data, which showed no significant difference in surface composition or regolith structure between the two lobes (Abe et al. 2006a; Saito et al. 2006). As discussed above, we have analysed this in detail and conclude that such a scenario can only realistically account for up to $\sim 5 \mathrm{~m}$ in the determined COM shift (Fig. 9).

b) Two completely unrelated bodies combined in a slow collision. The uniform surface composition and regolith structure strongly precludes this. We surmise that the probability must be negligibly low for two unrelated objects to come together 
at a sufficiently-low encounter velocity to ensure the survival of both lobes upon contact and preserve its "bi-lobed" shape, and have identical surface compositions and distinctive surface regolith structure.

c) The "head" and "body" formed from the remains of a catastrophic collision on a larger differentiated body, presumably in the main asteroid belt. While this could certainly produce two bodies of very different bulk densities, which later came together, the same principle applies as in scenario "b", at least to a certain extent as we shall discuss. If a high-density fragment from the inner core of the original body settled on the surface of what is now the "body" of Itokawa, then the fragment would subsequently need to be completely enveloped in material identical in composition to the "body", and develop a similar regolith structure. Alternatively, the "head" could be predominantly a monolithic fragment of the same material, but with higher density than the porous rubble pile "body". In this case less processing of the surface of the "head" would be required to disguise its nature. There are various processes that could alter an asteroid's surface, although not all have been confirmed by observations. They include space weathering, collisions, gravitational torques and tidal forces, YORP torques and possibly YORP-induced "seismic shaking". However, each of these processes may affect each lobe in different ways and so both lobes would need to have experienced precisely the right combination of evolutionary processes to end up identical, and leave no trace that the high-density fragment was present. Of course, we cannot rule out the possibility that the fragment is comprised of high-density metallic material, that was sufficiently small to be subsumed by the coalescing silicate material, and thus buried somewhere towards the "head" region. Michel \& Richardson (2013) examined the impact scenario using an $\mathrm{N}$-body simulation, resulting in a body with multiple attaching relatively-large lobes. But there are a number of issues that preclude conclusive comparison with Itokawa. Firstly the initial body is approximately $50 \times$ larger than Itokawa, with the largest remaining fragment being $\sim 40 \times$ larger. The results for a simulation using a much smaller body may be completely different. Furthermore, there are many large lobes produced in this simulation, rather than the simple "bilobed" structure observed. Nor are density inhomogeneities between lobes considered. With just one permutation being simulated, the probability of ending up with something that resembles Itokawa is unknown. On the other hand, the simulations do provide a means to produce the uniform surface composition and topography. It is clearly important to develop simulations of this kind, and reproduction of a bi-lobed Itokawa with the density disparity that we report represents a fascinating challenge for the modelling community.

d) A fourth scenario involves a single consolidated body that was spun-up by YORP, leading to the migration of regolith particles towards the equatorial region (Ostro et al. 2006; Scheeres et al. 2006). This material was eventually lifted off the surface, with the orbiting material coalescing into a satellite (Walsh et al. 2008). Both the process of regolith transport and ejection, and the re-aggregation and subsequent dynamics of the secondary, could alter the densities of both primary and secondary. In the case of the (66391) 1999 KW4 binary system, which shows similar density differences between both components of the system (Ostro et al. 2006), it has been proposed that the rapid spin rate of the primary leads to enhanced porosity and thus a lowering of its density (Scheeres et al. 2006). Conversely, porosity is reduced in the secondary due to dynamical instabilities leading to periodic "shaking", thereby increasing the density of the secondary. This scenario, or some variation of it, is certainly feasible for the "head" of Itokawa. Although Itokawa's current long rotation period precludes porosity decrease from rapid spin rate, the density of the "body" will be determined by its original, very different formation environment (presumably involving the re-aggregation of fragments from a high-speed collision with another body), coupled with the residual effects of the earlier regolith-loss process. Once the binary system has stabilized, Binary YORP (or "BYORP") (Ćuk \& Burns 2005) could have caused the orbital semi-major axis to steadily evolve, slowly guiding the smaller secondary in towards the main primary body until it eventually made gentle contact and settled (although we note that BYORP is not strictly required for such a system to collapse - see Jacobson \& Scheeres 2011). Such "contact binaries" have been observed, with perhaps the most striking example being 1996 HW1 (Magri et al. 2011). Of course, the existing distribution of fine-grain regolith on Itokawa may be an important constraint for any modelling tests of this scenario.

Whichever scenario is correct, based on our measurement of a density inhomogeneity, we can now infer with a high degree of confidence that Itokawa formed from the merging of two separate asteroids, either in the aftermath of a catastrophic disruption of a larger differentiated body, or from the collapse of a binary system. We also successfully demonstrate that an observational measurement of radiative torques, when combined with a detailed spacecraft shape model, can provide insight into the interior structure of an asteroid.

Acknowledgements. We thank the anonymous referee for their helpful comments. We thank all the staff at the observatories involved in this study for their support. This work was based on observations at the following observatories: ESO, Chile (PID: 185.C-1033); The Liverpool Telescope, La Palma; Palomar Observatory, California; The Isaac Newton Group, La Palma; Steward Observatory, Arizona; Table Mountain Observatory, California. S.C.L., S.R.D., B.R., and S.F.G. gratefully acknowledge support from the UK Science and Technology Facilities Council. S.C.L. acknowledges support from the Southeast Physics Network (SEPnet). CS received funding from the European Union Seventh Framework Programme (FP7/2007-2013) under grant agreement No. 268421. This work was performed in part at the Jet Propulsion Laboratory under a contract with NASA. This work made use of the NASA/JPL HORIZONS ephemeris-generating programme. All image reduction and processing were performed using the Image Reduction and Analysis Facility (IRAF) (Tody $1986 \&$ 1993). IRAF is distributed by the National Optical Astronomy Observatories, which are operated by the Association of Universities for Research in Astronomy, Inc., under cooperative agreement with the National Science Foundation.

\section{References}

Abe, M., Takagi, Y., Kitazato, K., et al. 2006a, Science, 312, 1334 Abe, S., Mukai, T., Hirata, N., et al. 2006b, Science, 312, 1344 Benner, L. A. M., Ostro, S. J., Magri, C., et al. 2008, Icarus, 198, 294 Breiter, S., Bartczak, P., Czekaj, M., Oczujda, B., \& Vokrouhlický, D. 2009, A\&A, 507, 1073

Ćuk, M., \& Burns, J. A. 2005, Icarus, 176, 418

Demura, H., Kobayashi, S., Nemoto, E., et al. 2006, Science, 312, 1347

Durech, J., Vokrouhlický, D., Kaasalainen, M., et al. 2008a, A\&A, 489, L25 Ďrech, J., Vokrouhlický, D., Kaasalainen, M., et al. 2008b, A\&A, 488, 345 Durech, J., Vokrouhlický, D., Baransky, A. R., et al. 2012, A\&A, 547, A10 Gaskell, R. 2008, Gaskell Itokawa Shape Model V1.0. HAY-A-AMICA-5ITOKAWASHAPE-V1.0, NASA Planetary Data System

Hapke, B. 1981, J. Geophys. Res., 86, 3039

Hapke, B., \& Wells, E. 1981, J. Geophys. Res., 86, 3055

Jacobson, S. A., \& Scheeres, D. J. 2011, Icarus, 214, 161

Kaasalainen, M., Torppa, J., \& Muinonen, K. 2001, Icarus, 153, 37

Kaasalainen, M., Durech, J., Warner, B. D., et al. 2007, Nature, 446, 420 
S. C. Lowry et al.: YORP spin-up detected on asteroid Itokawa

Lowry, S. C., Fitzsimmons, A., Pravec, P., et al. 2007, Science, 316, 272 Magri, C., Howell, E. S., Nolan, M. C., et al. 2011, Icarus, 214, 210

Michel, P., \& Richardson, D. C. 2013, A\&A, 554, L1

Müller, T. G., Sekiguchi, T., Kaasalainen, M., et al. 2005, A\&A, 443, 347

Nolan, M. C., Magri, C., Howell, E. S., et al. 2013, Icarus, 226, 629

Ostro, S. J., Hudson, R. S., Benner, L. A. M., et al. 2002. In Asteroids III, eds. W. F. Bottke Jr., A. Cellino, P. Paolicchi, \& R. P. Binzel (Tucson: University of Arizona Press), 151

Ostro, S. J., Benner, L. A. M., Nolan, M. C., et al. 2004, Meteor. Planet. Sci., 39 , 407

Ostro, S. J., Margot, J.-L., Benner, L. A. M., et al. 2006, Science, 314, 1276

Pravec, P., Vokrouhlický, D., Polishook, D., et al. 2010, Nature, 466, 1085

Rozitis, B., \& Green, S. F. 2011, MNRAS, 415, 2042

Rozitis, B., \& Green, S. F. 2012, MNRAS, 423, 367

Rozitis, B., \& Green, S. F. 2013, MNRAS, 433, 603

Rubincam, D. P. 2000, Icarus, 148,2
Saito, J., Miyamoto, H., Nakamura, R., et al. 2006, Science, 312, 1341 Scheeres, D. J., \& Gaskell, R. W. 2008, Icarus, 198, 125

Scheeres, D. J., Fahnestock, E. G., Ostro, S. J., et al. 2006, Science, 314 1280

Scheeres, D. J., Abe, M., Yoshikawa, M., et al. 2007, Icarus, 188, 425

Slivan, S. M. 2002, Nature, 419, 49

Statler, T. S. 2009, Icarus, 202, 502

Taylor, P., Margot, J.-L., Vokrouhlický, D., et al. 2007, Science, 316, 274

Tody, D. 1986, in Instrumentation in Astronomy VI, ed. D. L. Crawford, Proc. SPIE, 627, 733

Tody, D. 1993, in Astronomical Data Analysis Software and Systems II, eds. R. J. Hanisch, R. J. V. Brissenden, \& J. Barnes, ASP Conf. Ser., 52, 173

Vokrouhlický, D., \& Nesvorný, D. 2008, AJ, 136, 280

Vokrouhlický, D., Nesvorny, D., \& Bottke, W. F. 2003, Nature, 425, 147

Walsh, K. J., Richardson, D. C., \& Michel, P. 2008, Nature, 454, 188 